\title{
MRI EVALUATION IN SUSPECTED CASES OF ROTATOR CUFF/ LABRAL TEARS ON LOW FIELD MRI SYSTEM
}

Pramod Shaha ${ }^{1}$, Nupoor Kothari², K. Sahoo ${ }^{3}$, Ravindra Gunaki ${ }^{4}$

HOW TO CITE THIS ARTICLE:

Pramod Shaha, Nupoor Kothari, K. Sahoo, Ravindra Gunaki. "MRI Evaluation in Suspected Cases of Rotator Cuff/ Labral Tears on Low Field MRI System". Journal of Evolution of Medical and Dental Sciences 2015; Vol. 4, Issue 15, February 19; Page: 2540-2552, DOI: 10.14260/jemds/2015/366

ABSTRACT: MRI is the 'Gold Standard' noninvasive procedure of choice for evaluation of diseases of the shoulder, especially with regard to identifying lesions of the rotator cuff and glenoid labrum. Low tesla MRI, being available at an affordable cost and with relatively low maintenance, suits to the requirement of the society in rural areas. This study was aimed at evaluating patients with suspected rotator cuff / labral tears on a Low field MRI system \& comparison of the imaging findings with arthroscopic and / or operative findings.

KEYWORDS: Low field MRI, Rotator cuff, glenoid labrum.

INTRODUCTION: Shoulder joint is a ball and socket type joint formed by articulation between head of humors and glenoid of scapula. Structurally it is a weak joint due to disparity of the articulating surface area between a small and shallow glenoid cavity in comparison to head of humerus. However this arrangement provides the glenohumeral joint with greater range of movements than any articulation in human body.

Large amount of clinical tests in physical examination are used for the diagnosis of painful shoulder for determining the exact site of periarticular lesions.

Clinical diagnosis have low accuracy in comparison with arthroscopy (gold standard, which is invasive. ${ }^{[1]}$

MRI is the 'Gold Standard' non-invasive procedure of choice for evaluation of diseases of the shoulder, especially with regard to identifying lesions of the rotator cuff and glenoid labrum \& is an excellent modality because of its multiplanar capability and soft tissue discrimination. ${ }^{[2]}$

The prevalence of rotator cuff tears in the general population has been found in cadaver and MRI studies to range from $5 \%$ to $39 \%$. Because the prevalence of rotator cuff disease increases with age, it is expected to become an even more common clinical condition as the population ages. ${ }^{[3]}$

There is no debate that high-field MR images are of superior diagnostic value compared to low-field images. Low-field MRI scanners, however, besides being available at a low cost, have open design, which is better tolerated by claustrophobic and pediatric patients. In the present setup, this helps in alleviating the anxiety in population of rural area thus facilitating patients' co-operation in the study.

A study conducted by Shellock et al. using a $0.2 \mathrm{~T}$ extremity-dedicated MR scanner, for the rotator cuff tears, shows good agreement of the low- field MR results with the surgical findings for determination of lesions of the rotator cuff and glenoid labrum. ${ }^{[2]}$

Ghazinoor and Crues (2007) stated that most studies that have been performed comparing high-field versus low-field MRI of the shoulder have demonstrated no significant difference in sensitivity and specificity in detection of rotator cuff and labral lesions. [3] 
This study carried out in a rural setting and was aimed at evaluating patients with suspected rotator cuff / labral tears on a Low field MRI system \& comparison of the findings with arthroscopic and / or operative findings.

MATERIALS AND METHODS: This was a prospective study, comprising of 56 patients who were referred for MRI of the shoulder joint with suspicion of Rotator cuff / Labral Tear. Patients who were contraindicated for MR imaging i.e., those having metallic implants; or patients who have had a history of surgery, infections or neoplasm in the shoulder joint to be imaged were excluded from the study.

The patients were scanned; with the arm in a mildly externally rotated position using 0.35 TESLA, SEIMENS, MAGNETOM C! Machine, using a 16 channel multipurpose coil. Sequences used were -Short T1 inversion recovery(STIR) in oblique coronal and sagittal planes; Proton density in oblique coronal and axial planes; T2 weighted images -oblique coronal plane, T2 weighted images axial plane andT2 weighted images - oblique sagittal plane,T1 weighted images in the oblique coronal plane and in oblique sagittal plane.

Comparison of MRI findings was done with arthroscopic / surgical findings. Arthroscopy findings were not considered in patients who underwent arthroscopy 60 days after the imaging was performed.

\section{Evaluation Criteria:}

\section{A.) Rotator Cuff Tears:}

1. Full thickness tears: An abnormality of the rotator cuff is considered a full thickness tear if it results in a connection between the articular and bursal surfaces of the cuff tendon.

The most specific sign of a full thickness Rotator Cuff tear is visualization of a complete defect in the tendon, extending from the articular surface completely through to the bursal surface. This defect is usually fluid signal intensity.

Secondary signs of a full thickness RC tear - Fluid in the sub acromial - sub deltoid bursa, muscle atrophy, intramuscular cysts, superior humeral migration, and retraction of the musculotendinous junction. $[2,4,5,6]$

2. Partial thickness tears: 3 Different types according to the portion of the tendon that is abnormal:

- Articular-sided tears.

- Bursal-sided tears, and

- Interstitial tears.

The combination of fluid, granulation tissue, and blood within partial thickness tears causes their hyperintense T2 signal characteristics, similar to those of full thickness tears, but without full thickness involvement of the tendon.

Tracking of fluid within articular / bursal surface is characteristic of partial tears on T2WI.

Interstitial tears, also known as intrasubstance tears or intramuscular cysts, can occur in isolation within the tendon without articular- or bursal- sided extension, or they can also occur in combination with either articular or bursal-sided partial thickness tears. $[4,5,6]$

3. Tendinosis: Histological changes of Rotator Cuff tendons, most commonly mucoid degeneration and fibro-cartilaginous metaplasia, appear as moderately increased focal irregular or diffuse intermediate intra-substance signal intensity on T1- and T2-weighted images. 
This altered MRI signal intensity does not rise to the same T2 signal intensity as fluid and is usually more globular in appearance and less linear than signal abnormalities seen with Rotator Cuff tears.

The tendon may be normal in caliber or demonstrate diffuse or focal thickening.

In practice, it can occasionally be difficult to distinguish between severe tendinosis and a partial thickness tear; sometimes both possibilities must be considered.[2,4,5]

4. Humeral head Cysts: Humeral head "cysts," foci of fluid signal intensity in the bone marrow of the greater tuberosity near the Rotator Cuff insertions.

\section{Muscle Abnormalities:}

Fatty Degeneration: Presence of fatty infiltration or muscle volume atrophy is best evaluated on the T1-weighted sagittal oblique images:

- No fatty deposits is described as no. fatty infiltration.

- A few fatty streaks, as minimal fatty infiltration.

- Some fatty infiltration, though still with more muscle than fat, as mild fatty infiltration.

- A roughly equal proportion of muscle and fatty infiltration as moderate fatty infiltration.

- Predominance of fatty infiltration, as advanced fatty infiltration.

Muscle Edema: Sagittal oblique T2- weighted images are used to carefully evaluate the muscle bellies for often subtle or asymmetric edema. Muscular edema often represents an acute injury, such as muscle strain, particularly within the clinical context of an acute injury and when correlating findings of an acute Rotator Cuff tear are present.

Neurogenic abnormalities can also cause muscle edema, if detected early, and muscle atrophy, if more chronic.

\section{Bursal Fluid:}

- Subacromial - Subdeltoid Bursa.

- Subscapularis Bursa.

\section{Degenerative / Bony Changes:}

- Acromion type.

- Acromio - Clavicular Jointarthrosis.

- Osteoarthritis.

- Fractures- Humerus / Scapula.

\section{Bicepstenosynovitis / Peribicipitaltendon Fluid:}

B.) LABRAL TEARS: The Glenoid Labrum is best seen on Axial T2WI.

MRI criteria for diagnosing labral tears include surface irregularity, increased signal within the substance of the labrum that extends to the labral surface (L), fluid or contrast imbibed into the substance of the labrum (M), and labral avulsions. Secondary signs of labral tears include paralabral cysts $(\mathrm{N})$, periosteal stripping and tearing, labral associated bone injuries such as Hill Sachs and Osseous Bankart lesions. 
Images were analyzed for the presence or absence of superior labral antero-posterior tears (SLAP tears), and anterior or posterior labral tears.

STATISTICAL METHODS: The standard definitions for sensitivity, specificity, positive predictive value, and negative predictive value were applied to the results of this study with regard to the use of MRI for diagnosis of a rotator cuff tear and/or labral lesion, with the surgical findings used as the gold standard.

RESULTS: In our study, out of the 56 patients who were evaluated, $43 \%$ of the patients were in the range of $20-40$ years, $52 \%$ of the patients were older than 40 years. Only $5 \%$ patients were less than 20 years old.

Males comprised $70 \%$ of the patients and the most commonly affected shoulder was the right shoulder, involved in $70 \%$ cases.

Out of the rotator cuff musculature, Supraspinatus was the most commonly affected muscle, with 6 cases demonstrating full thickness tears, 13 cases with partial thickness tears and 14 cases with tendinosis.

Infraspinatus was the next most commonly affected muscle with 2 cases demonstrating full thickness tears, 6 cases demonstrating partial thickness tears and 8 cases with tendinosis.

Teres Minor and Subscapularis were rarely involved with 1 case demonstrating tendinosis of the teres minor and 1 case demonstrating partial thickness tear of the subscapularis.

Of the 6 cases of supraspinatus full thickness tears, 2 demonstrated Grade III tendon retraction and 3 demonstrated Grade II retraction.

1 case of full thickness infraspinatus tear also showed Grade III tendon retraction.

There was evidence of fatty atrophy in 5 cases and muscle edema in 2 cases of patients with full thickness and partial thickness tears, occurring more in patients with full thickness tears.

Subacromial - subdeltoidand subscapular bursal fluid was seen in all patients of full thickness tears and in all cases of partial thickness tears except for 2 cases.

The relative occurrence of fluid in the abovementioned bursae was significantly lower in cases of tendinosis.

However, Subacromial -subdeltoidbursal fluid was absent in almost all normal cases except 1 and 7 normal cases had evidence of fluid in the subscapular bursa.

No correlation could be established between the occurrence of humeral head cysts and rotator cuff tears as there was evidence of cysts in only 1 case of full thickness tears, 3 cases of partial thickness tears and cysts were also present in 6 normal cases.

Peri-bicipital tendon fluid had a higher incidence in cases of full thickness tears $(n=63 \%)$ and partial thickness tears $(n=50 \%)$ as compared to cases of tendinosis and patients with no abnormality.

8 out of the 56 patients had imaging findings consistent with Labral tears.

The anterior labrum was most commonly afflicted, seen to be involved in 5 cases with 4 of them having a Bankart lesion (Antero-inferior labral tear).

The posterior labrum and the Superior labrum were seen to be affected in 1 and 2 cases respectively.

Arthroscopic results were available for 50 patients out of the sample size of 56. 
6 patients had findings confirmed on surgery.

Full thickness tears of the supraspinatus and infraspinatus had a sensitivity and specificity of $100 \%, 100 \%$ \& 67\%, 100\% on arthroscopic correlation respectively.

Partial thickness tears of the supraspinatus had a sensitivity, specificity, positive predictive value, negative predictive value of 75\%, 90\%, $69 \%, 93 \%$ respectively.

Partial thickness tears of the infraspinatus had a sensitivity, specificity, positive predictive value, negative predictive value of 50\%, 94\%, 50\%, 94\% respectively.

Compared to arthroscopic findings, anterior labral tears had good correlation in that 4 out of the 5 diagnosed tears were confirmed on surgery.

Similarly, all the posterior labral and SLAP tears were confirmed on arthroscopy/surgery.

DISCUSSION: MRI has been reported to be a particularly effective imaging modality for evaluation of full- thickness rotator cuff tears, with partial tears being somewhat more difficult to reliably detect.

It is particularly useful in detecting soft tissue damage or disease. The quality of MR images depends not only upon field strength (above 1 Tesla (T) is considered high), but also coil selection, contrast administration, imaging plane and sequence parameters, and ultimately interpreter experience and familiarity with pathologic processes and surgical interventions.

In this study, 56 patients with suspected rotator cuff / labral lesions were evaluated and imaging findings were correlated with arthroscopic / surgical findings to assess accuracy of the same.

Pathological findings were most commonly seen in the age group older than 40 (52\%), followed by 20-40 year old patients (43\%). The least incidence of such pathologies was in the age group less than 20 years ( $5 \%$ ).

This correlates well with previous studies of an increased incidence of cuff tears in patients older than 40 years than younger patients indicating a degenerative component of the cuff predisposing to tears. ${ }^{[3,7]}$

However, this was inferred from asymptomatic patients in western populations and hence cannot be compared to our study population most of whom presented with a positive history of trauma, and performed jobs requiring a greater level of physical activity.

Males were commonly affected (70\%), likely indicating association with increased level of physical activity, trauma.

Supraspinatus was the most commonly affected muscle, with 6 cases demonstrating full thickness tears followed by infraspinatus in which 2 cases demonstrated full thickness tears. Teres Minor and Subscapularis were rarely involved. Individual cuff component muscle / tendon incidences have not been provided/ evaluated in the previously mentioned studies; hence a comparison could not be done.

There was an increased incidence of fatty atrophy and muscle edema in patients with rotator cuff tears, more so, in patients with full thickness tears.

Sub acromial - sub deltoid and subscapular bursal fluid was seen in all patients of full thickness tears and in all cases of partial thickness tears except for 2 cases, indicating a positive correlation with cuff pathology. Seven normal cases had evidence of fluid in the subscapular bursa, and one of the normal cases had evidence of fluid in the sub acromial - sub deltoid bursa, indicating that fluid in this bursa imparts more diagnostic importance to cuff tears than the subscapular is 
bursa. These findings parallel the previously established criteria of fluid in the Sub acromial- Sub deltoid bursa, being a secondary sign of full thickness rotator cuff tears. ${ }^{[8,9]}$

Peribicipital tendon fluid and humeral head cysts did not correlate well with rotator cuff pathololgy, though there was an increased incidence of peribicipital tendon fluid in patients with full thickness (63\%) and partial thickness tears (50\%).

8 out of the 56 patients had imaging findings consistent with Labral tears. The anterior labrum was most commonly afflicted, seen to be involved in 5 cases with 4 of them having a Bankart lesion (Antero-inferior labral tear). The posterior labrum and the Superior labrum were seen to be affected in 1 and 2 cases respectively. This was consistent with the study by Magee et al. (2003) [10], having 2 anterior labral tears and one SLAP lesion, in that anterior labral tears were the commonest.

Full thickness tears of the supraspinatus had a sensitivity, specificity, postive predictive value and negative predictive value of $100 \%, 100 \%, 100 \%, 100 \%$ on arthroscopic/surgical correlation respectively. Similar statistical indices for infraspinatus tears were $67 \%, 100 \%, 100 \%$, 98\% respectively.

Partial thickness tears of the supraspinatus had sensitivity, specificity of $75 \%, 90 \%$, with sensitivity of $50 \%$ \& specificity of $94 \%$ for infraspinatus. This was consistent with previous study by Joseph P. Iannotti et al (1991) [11] where Magnetic resonance imaging demonstrated 100 per cent sensitivity and 95 percent specificity in the diagnosis of complete tears.

In the study by Zlatkin et al. (2004) for the rotator cuff tears, where the sensitivity, specificity, positive predictive value, and negative predictive value were 90\%, 93\%, 98\%, and 68\% respectively, our study shows comparative results. ${ }^{[4]}$

In a similar study by Shellock et al. (2001),[2] to detect lesions of the rotator cuff and glenoid labrum in 47 patients using a $0.2 \mathrm{~T}$ scanner, for the rotator cuff tears (combined full thickness and partial thickness), the sensitivity, specificity, positive predictive value, and negative predictive value were $89 \%, 100 \%, 100 \%$, and $90 \%$ respectively, again revealing good correlation to our study.

The three false negatives encountered were related to partial thickness tears.

However, a study by Magee et al. (2003) ${ }^{[10]}$ used a 0.2 T open-design MR scanner for detection of supraspinatus tendon tears and labral tears in 40 patients and in four patients, fullthickness supraspinatus tendon tears, seen on arthroscopy, could not be depicted.

Such a false negative result for full thickness supraspinatus tear was not seen in our study.

This can be attributed to the superior imaging quality of the present day 0.35T unit with multichannel incorporation, as compared to the MRI system in the abovementioned study.

Previously reported sensitivities and specificities for detection of partial tears ranged from 0\%- $92 \%$ and $85 \%-99 \%$, respectively.

Although the statistical range coincides with our study, however, these prior studies were conducted using mid- and high-field- strength, whole-body MR systems.[10]

Partial tears are known to be difficult to visualize on MRI because their depth may be shallow and the associated abnormal signal intensity may be indistinguishable from that which occurs with degeneration, tendinitis, or tendinopathy.

The similarity and overlap of signal patterns for these lesions generally creates interpretation problems regardless of the field strength of the MR system, the shoulder coils, or the pulse sequences used for imaging. $[5,12,13,14]$ 
In the study by Zlatkin et al. (2004),[4] for the labrum, the sensitivity, specificity, positive predictive value, and negative predictive value were $55 \%, 100 \%, 100 \%$, and $82 \%$, respectively.

Shellock et al. (2001) ${ }^{[2]}$ found that, for labral lesions, the sensitivity, specificity, positive predictive value, and negative predictive value were $89 \%, 95 \%, 80 \%$, and 97\% (two false positives, one false negative), respectively.

Similarly, Allmann et al. (1998)[15] used a 0.2 T MR scanner to determine the diagnostic accuracy of glenohumeral instabilities with low-field MRI. The sensitivity, specificity for $0.2 \mathrm{~T}$ MR was $91 \%$ \& $67 \%$ respectively for labral pathology.

These studies were fairly consistent with our study in that there was a high arthroscopic / surgical correlation of the labral pathologies, though our low sample size in labral tears was a limitation in having a more accurate representation.

Four patients in our study, all within the 15-30 year age group, presented with a clinical suspicion of cuff pathology, however had imaging findings consistent with edema in the supraspinatus and infraspinatus muscle bellies, with no evidence of tear / tendinitis, indicating likely suprascapular nerve denervation, which can be labeled a 'clinical mimic' of cuff tears.

One patient, aged 40 years, had calcific foci in the infraspinatus tendon, near its humeral insertion, however no prior / recent history of trauma, validated a diagnosis of calcific tendinitis.

LIMITATIONS OF THE STUDY: Firstly, similar to previous studies, it was a study that included symptomatic patients (Based on a thorough clinical examination by the referring surgeon).

Therefore, bias was introduced in favor of diagnosing rotator cuff and labral lesions on MRI, even though we were unaware of the final surgical findings.

Secondly, there was a variable interval between imaging and surgery, ranging from 8 to 42 days. The length of this interval can be attributed to the amount of time necessary for the formulation, acceptance and implementation of a prescribed treatment plan, either conservative or surgical.

This interval can result in both, either a progression of the pathology or regression, from that encountered at the time of imaging, hence confounding the correlation.

Third, the use of arthroscopic procedures as a gold standard of reference may be a weakness because there may be surgeon-dependent variations, and more importantly, subtle findings may escape arthroscopic visualization.

Furthermore, some arthroscopists routinely explore the sub- acromial space, while others do not.

Such problems of visualization may be compounded by the presence of synovitis and marked degeneration of the glenohumeral joint, as well as bursitis and marked degeneration affecting the subacromial space.

CONCLUSION: This prospective study aimed at evaluating rotator cuff and labral tears in symptomatic individuals using a $0.35 \mathrm{~T}$ low field strength scanner.

Total 56 patients mostly low socio-economic status were included in the study, and followed up subsequently for comparison of the imaging findings with arthroscopic / surgical findings. 
MAGNETIC RESONANCE IMAGING is the 'gold standard' noninvasive procedure of choice for evaluation of diseases of the shoulder, especially with regard to identifying lesions of the rotator cuff and glenoid labrum thus facilitating the selection of an appropriate treatment plan.

The choice of a low - field strength scanner in our study was apt for the study population, a major concern for whom was cost effectiveness.

Our study demonstrates an excellent statistical accuracy of full thickness cuff tears with 'gold standard' arthroscopic / surgical findings. This statistical accuracy is diminished in partial thickness tears and in tendinopathy.

Labral tears, although hampered by a small sample size, however demonstrated a good statistical arthroscopic correlation.

It is crucial to differentiate between full-thickness and partial-thickness tears because the treatments for these rotator cuff lesions differ. A full-thickness tear typically requires surgery.

In conclusion, the findings of this study indicated that shoulder MRI performed with a lowfield extremity MR system exhibited acceptable diagnostic accuracy in depicting lesions of the rotator cuff and labrum based on a comparison between the MR results and surgical findings.

The overall diagnostic capabilities were comparable to those reported in the peer-reviewed literature for the use of whole-body and mid- and high-field MR systems, establishing a low -field scanner as a reliable alternative at centres, where, due to the socio-economic status of the population being investigated, cost effectiveness is a major determining factor.

\section{BIBLIOGRAPHY:}

1. Nørregaard, M R Krogsgaard, T Lorenzen, E M Jensen. Diagnosing patients with longstanding shoulder joint pain; Ann Rheum Dis 2002; 61: 646-649.

2. Shellock FG, Bert JM, Fritts HM, Gundry CR, Easton R, Crues JV 3rd. Evaluation of the rotator cuff and glenoid labrum using a 0.2-Tesla extremity magnetic resonance (MR) system: MR results compared to surgical findings; J MagnReson Imaging. 2001 Dec; 14(6): 763-70.

3. Karen P. Barr, MD. Rotator cuff disease; Phys Med RehabilClin N Am 15 (2004) 475-491.

4. Zlatkin MB, Hoffman C, Shellock FG. Assessment of the rotator cuff and glenoid labrum using an extremity MR system: MR results compared to surgical findings from a multi-center study. J MagnReson Imaging. 2004 May; 19(5): 623-31. Olivier P. Krief, Dominique Huguet. Shoulder Pain and Disability: Comparison with MR Findings; AJR 2006; 186: 1234-1239.

5. Rafii M, Firooznia H, Sherman O, et al. Rotator cuff lesions: signal patterns at MR imaging. Radiology 1990; 177: 817-823.

6. Farley TE, Neumann CH, Steinbach LS, Jahnke AJ, Petersen SS. Full- thickness tears of the rotator cuff of the shoulder: diagnosis with MR imaging. AJR Am J Roentgenol 1992; 158: 347351.

7. JS Sher; JW Uribe; A Posada; BJ Murphy; MB Zlatkin. Abnormal findings on magnetic resonance images of asymptomatic shoulders; J Bone Joint Surg Am, 1995 Jan 01; 77(1): 10-15.

8. Clyde A. Helms. Magnetic Resonance Imaging of the Shoulder, Fundamentals of Skeletal Radiology 3rd edition: $184-194$.

9. Stoller DW, Fritz RC. Magnetic resonance imaging of impingement and rotator cuff tears. MagnReson Imaging Clin N Am 1993; 1: 47-63. 


\section{ORIGINAL ARTICLE}

10. Magee T, Shapiro M, Williams D. Comparison of high-field-strength versus low-field-strength MRI of the shoulder; AJR Am J Roentgenol. 2003 Nov; 181(5): 1211-5.

11. JP Iannotti, MB Zlatkin, JL Esterhai, HY Kressel, MK Dalinka, KP Spindler. Magnetic resonance imaging of the shoulder; J Bone Joint Surg Am, 1991 Jan 01; 73(1): 17-29.

12. Balich SM, Sheley RC, Brown TR, Sauser DD, Quinn SF. MR imaging of the rotator cuff tendon: interobserver agreement and analysis of interpretive errors. Radiology 1997; 204: 191-194.

13. Wnorowski DC, Levinsohn EM, and Chamberlain BC, McAndrew DL. Magnetic resonance imaging assessment of the rotator cuff: is it really accurate? Arthroscopy 1997; 13: 710-719.

14. Shellock FG, Stoller D, Crues JV. MRI of the shoulder. A rational approach to the reporting of findings. J Magn Reson Imaging 1996; 6: 268-270.

15. Allmann KH, Walter O, Laubenberger J, Uhl M, Buitrago-Tellez CH, Biebow N, et al. Magnetic resonance diagnosis of the anterior labrum and capsule. Effect of field strength on efficacy. Invest Radiol. 1998 Jul; 33(7): 415-20.



Fig. 1

Coronal STIR image demonstrating Full Thickness Supraspinatus tendons tear with tendon retraction.



Fig. 2

Coronal STIR image showing altered signal intensity in the supraspinatus tendon near its insertion with disruption of fibres near articular surface, indicating partial tear. 


\section{ORIGINAL ARTICLE}

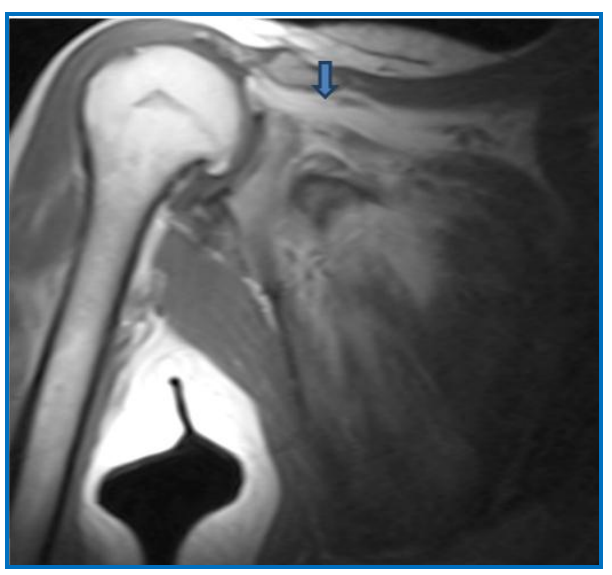

\section{Fig. 3}

Coronal T1WI, showing hyperintensity in the supraspinatus muscle indicating fatty atrophy.



Fig. 4

Coronal STIR image demonstrating a SLAP tear.

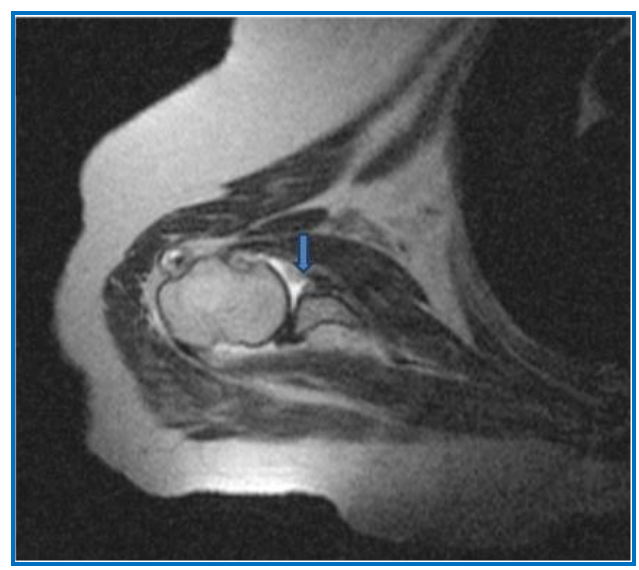

\section{Fig. 5}

Axial STIR image showing a tear of the anterior labrum. 


\section{ORIGINAL ARTICLE}

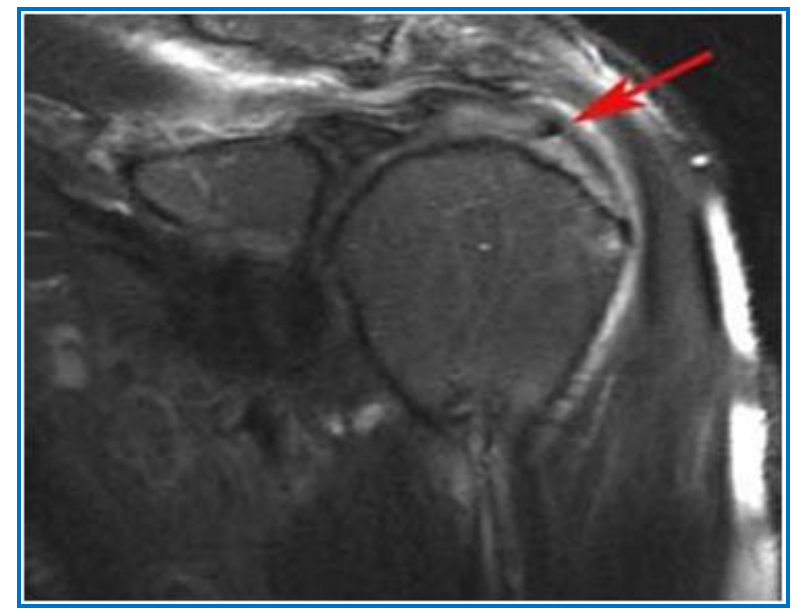

Fig. 6

MRI image of a partially torn rotator cuff. The red arrow marks the torn edge of the rotator cuff, note the wavy tendon edge



Fig. 7

The typical appearance of the torn cuff in the above patient as viewed through the arthroscope. 


\section{ORIGINAL ARTICLE}

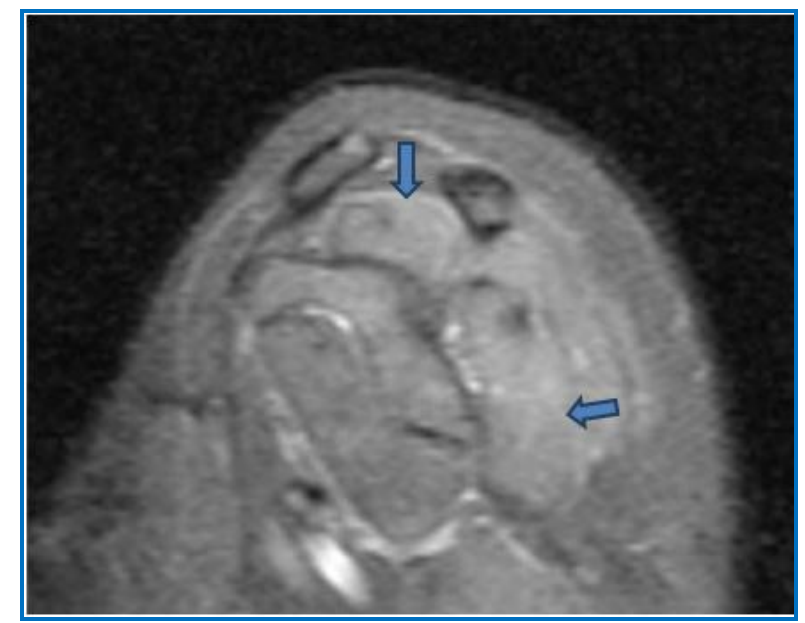

\section{Fig. 8}

STIR Sagittal image showing hyperintensity in supraspinatus and infraspinatus muscles indicating edema, as a sequelae to suprascapular nerve compression.



\section{Fig. 9}

Axial T2WI, showing hypointensity, consistent with calcification, adjacent to the insertion of the infraspinatus tendon on the humeral head indicating calcific tendinitis. 


\section{ORIGINAL ARTICLE}

\section{AUTHORS:}

1. Pramod Shaha

2. Nupoor Kothari

3. K. Sahoo

4. Ravindra Gunaki

\section{PARTICULARS OF CONTRIBUTORS:}

1. Professor, Department of Radiodiagnosis, KIMS, Karad.

2. Resident, Department of Radiodiagnosis, KIMS, Karad.

3. Professor \& HOD, Department of Radiodiagnosis, KIMS, Karad.

FINANCIAL OR OTHER COMPETING INTERESTS: None
4. Professor, Department of Orthopaedics, KIMS, Karad.

\section{NAME ADDRESS EMAIL ID OF THE} CORRESPONDING AUTHOR:

Dr. Nupoor Kothari, Department of Radiodiagnosis, Krishna Institute of Medical Sciences, Karad District, Satara-415110.

E-mail: drnupur@ymail.com

Date of Submission: 22/01/2015. Date of Peer Review: 23/01/2015. Date of Acceptance: 10/02/2015. Date of Publishing: 18/02/2015. 